\section{Azacitidine as salvage therapy for acute myeloid leukemia in a severely ill patient}

\author{
Harry Ross Powers, ${ }^{1}$ Moshe Bachar, ${ }^{1}$ \\ Natasha Savage, 2,3 Michael Toscano, ${ }^{2}$ \\ Paul M. Dainer ${ }^{3,4}$ \\ Departments of ${ }^{1}$ Medicine and \\ 2Pathology, Medical College of Georgia, \\ Georgia Regents University, Augusta, GA; \\ ${ }^{3}$ Charlie Norwood Veteran Affairs \\ Medical Center, Augusta, GA; \\ 4Section of Hematology/Oncology, \\ Medical College of Georgia, Georgia \\ Regents University, Augusta, GA, USA
}

\begin{abstract}
Acute myeloid leukemia (AML) is a hematological malignancy of myeloid progenitor cells that disrupt normal hematopoiesis. Current chemotherapy regimens result in complete remission in many cases; however, there exists no standard efficacious therapy for refractory acute myeloid leukemia. The hypomethylating agent, azacitidine, is effective in a limited number of such cases. We present a 57-year-old Filipino male with acute myeloid leukemia who was refractory to two induction chemotherapy regimens; however, he achieved complete remission after palliative therapy with azacitidine. We report this case to demonstrate the efficacy of azacitidine in refractory acute myeloid leukemia. Although the effectiveness of azacitidine in improving overall survival has been shown, this case demonstrates the effect on remission induction in high risk AML. Further studies are needed to delineate subsets of acute myeloid leukemia in which azacitidine will serve as effective therapy and to identify other targeted agents that may potentiate its effects.
\end{abstract}

\section{Introduction}

Acute myeloid leukemia (AML) is a clonal, hematological malignancy of myeloid progenitor cells. These progenitor blast cells accumulate in the bone marrow and peripheral blood, eventually disrupting normal hematopoiesis and leading clinically to anemia, neutropenia and thrombocytopenia. ${ }^{1}$ The prognosis for AML is variable, and reflects a number of clinical and genetic factors. ${ }^{2}$

Currently, AML is considered treatable and curative in many cases with a remissioninduction chemotherapy regimen followed by consolidative chemotherapy and, in many cases, hematopoietic stem cell transplant in first or subsequent remissions. In 1973, a standard regimen of 7 days of continuous infusion cytarabine and 3 days of daunorubicin was established. The initial remission rates have varied from $55-90 \%$ of patients after $7+3$ therapy in patient cohorts with a median age of $50 ; 3,4$ however, $20-30 \%$ of young patients and $40-50 \%$ of older patients will experience primary induction failure. ${ }^{5}$ The 5 -year survival rate for recurrent AML is only $11 \%{ }^{2}$ There still remains considerable variability in assigning appropriate treatment for primary-refractory and recurrent leukemia. ${ }^{6}$

Azacitidine is a hypomethylating agent shown to be more effective compared to best support care in elderly patients or patients with poor performance state and serious comorbidities. ${ }^{7}$ Azacitidine acts by inhibiting DNA methyltranferase once it is incorporated in the growing DNA strand. ${ }^{8}$ The drug's limited efficacy in AML was demonstrated as far back as the 1970's. ${ }^{9}$ Nevertheless, it did not become commercially available until 2004 when approved as the first drug for the treatment of myelodysplastic syndromes (MDS). Renewed interest in its potential to treat AML quickly followed. Several additional clinical studies have demonstrated its efficacy in treating relapsed or refractory AML. ${ }^{6,10,11}$ We recently treated a patient whose case illuminates the potential of azacitidine therapy in refractory AML with monocytic features under severely adverse conditions.

\section{Case Report}

A 57-year-old Filipino man presented with de novo AML, hyperuricemia, fulminant acute renal failure with a creatinine of $9.9 \mathrm{mg} / \mathrm{dL}$ and leukocytosis $\left(80.6 \times 10^{3} / \mathrm{mm}^{3}\right)$, features associated with an increased risk of early death. ${ }^{12} \mathrm{He}$ initially presented to the Emergency Department complaining of progressive dyspnea, anorexia and a 20-pound weight loss over the prior three to four weeks. His past medical history was significant for mitral valve replacement for mitral valve prolapse, seizure disorder, hyperlipidemia, cigarette smoking and heavy alcohol use. He denied fever, night sweats, pruritus, rash, or cough. His family history was non-contributory. His vital signs and body temperature were normal. Physical exam was significant for a grade II/VI systolic ejection murmur in left upper sternal border, bibasilar crackles on lung auscultation and trace pretibial edema. No lymphadenopathy or hepatosplenomegaly were noted. His admission $\mathrm{CBC}$ was significant for a white blood cell count (WBC) of $80.6 \times 10^{3} / \mathrm{mm}^{3}$ with differential of $13 \%$ segmented neutrophils, $3 \%$ bands,
Correspondence: Paul M. Dainer, Section of Hematology/Oncology, Medical College of Georgia, Georgia Regents University, BAA-5407, 1120 15th Street, Augusta, GA 30912-3125, USA. Tel.: +1.706.721.2505 - Fax: +1.706.721.5566. E-mail: pdainer@gru.edu

Key words: leukemia, acute myeloblastic/monocytic/refractory, azacitidine, salvage chemotherapy.

Acknowledgments: the authors would like to thank Dr. Jeremy Pantin for his editorial suggestions. This material is the result of work supported with resources and the use of facilities at the Charlie Norwood Veterans Affairs Medical Center, Augusta, Georgia. The contents do not represent the views of the Department of Veterans Affairs nor the United States Government.

Contributions: the authors contributed equally.

Conflicts of interest: the authors declare no potential conflicts of interest.

Received for publication: 12 June 2014.

Revision received: 6 August 2014.

Accepted for publication: 19 August 2014.

This work is licensed under a Creative Commons Attribution NonCommercial 3.0 License (CC BYNC 3.0).

(C) Copyright H.R. Powers et al., 2014

Licensee PAGEPress, Italy

Hematology Reports 2014; 6:5516

doi:10.4081/hr.2014.5516

$4 \%$ lymphocytes, and 75\% monocytes with many immature forms and a platelet count of $88 \times 10^{3} / \mathrm{mm}^{3}$. These results represent a marked change from his $\mathrm{CBC}$ done only four months previously. At that time, his $\mathrm{CBC}$ demonstrated a WBC of $7.9 \times 10^{3} / \mathrm{mm}^{3}$ with a normal differential, a platelet count of $150 \times 10^{3} / \mathrm{mm}^{3}$ and a hemoglobin of $14.6 \mathrm{~g} / \mathrm{dL}$. Bone marrow aspiration with flow cytometry, cytogenetics and fluorescent in-situ hybridization (FISH) studies demonstrated acute myeloid leukemia, WHO classification not otherwise specified (with monocytic differentiation), with $84 \%$ blasts, $3 \%$ segmented neutrophils, $2 \%$ nucleated red blood cells, $3 \%$ lymphocytes and $5 \%$ monocytes. Cytogenetic studies revealed a normal karyotype and FISH demonstrated no mutations (5q12, 5q31, 7cen, $7 \mathrm{q} 31.8$ cen, and $20 \mathrm{q} 12$ ) commonly observed in AML or MDS. JAK2 mutational studies were likewise negative. He received rasburicase for hyperuricemia and underwent acute hemodialysis. His hyperuricemia and acute renal failure resolved.

The patient initially received non-anthracycline based induction therapy with fludarabine (15 mg/m² IV BID for 4 days) and cytarabine 
( $500 \mathrm{mg} / \mathrm{m}^{2}$ IV BID for 4 days) because echocardiography had demonstrated decreased cardiac contractility and an ejection fraction (EF) of $40-45 \%$. After the first induction, his bone marrow remained hypercellular with $94 \%$ monoblasts and a normal karyotype. There were no mutations commonly seen in AML (FISH negative for MLL, BCR-ABL1, PDGRA, and PDGRB rearrangements), and atypical megakaryocytes that were not present on the initial diagnostic marrow. A MUGA later demonstrated an $\mathrm{EF}$ of $64 \%$. He was re-induced with infusional cytarabine $(100 \mathrm{mg} / \mathrm{mg}$ CIVI $\mathrm{d} 1-7)$ and daunorubicin (60 mg/m2/d IV d1-3). After his second induction course, the day 15 bone marrow continued to demonstrate $22 \%$ monoblasts with a normal karyotype, no common MDS mutations seen by FISH and megakaryocytes with atypia. The patient failed to achieve a complete remission after these two cycles of induction chemotherapy. Within 16 days following completion of the second induction, his leukocyte count rose again to greater than $100 \times 10^{3} / \mathrm{mm}^{3}$ with $30 \%$ segmented neutrophils, $17 \%$ monocytes, and $53 \%$ blasts and platelets rose higher than $500 \times 10^{3} / \mathrm{mm}^{3}$ (Figure 1). Hyper-leukocytosis was managed briefly with hydroxyurea, followed by azacitidine $\left(75 \mathrm{mg} / \mathrm{m}^{2}\right)$ subcutaneously for 7 -days every 28 days. His blood counts rapidly normalized. Following 5 cycles of azacitidine, a bone marrow aspirate and biopsy demonstrated a morphologic complete response (CR) with $0 \%$ blasts, $11.5 \%$ myelocytes, $10.5 \%$ metamyelo- cytes, $18.5 \%$ segmented neutrophils, $47.5 \%$ erythroid precursors, 4\% lymphocytes, $1 \%$ monocytes, $4 \%$ eosinophils, and 1\% basophils with normal cytogenetics and FISH. He is currently continuing azacitidine maintenance therapy and remains in complete remission after ten additional cycles.

\section{Discussion and Conclusions}

Response of refractory AML to azacitidine has been documented as far back as the 1970 s. $^{8}$ A recent clinical study assessed the efficacy of hypomethylating agents (azacitidine and decitabine) as induction, salvage, or consolidation therapy for AML. ${ }^{9}$ They reported that hypomethylating agents used as induction therapy resulted in a response rate of $26 \%$; however, as salvage therapy, only one of 28 patients achieved a CR. In another study, $21 \%$ of patients treated with azacitidine for recurrent or refractory disease achieved a CR. Of this group, six patients later underwent a hematopoietic stem cell transplant (HSCT). ${ }^{6}$ Both studies showed a similar median overall survival (OS) of the whole cohort of 8 and 9 months, respectively; however, among the patients that achieved a CR, median OS was not reached. These results suggest that the complete responses achieved may be durable. Furthermore, a French study of 141 patients with refractory AML after intensive

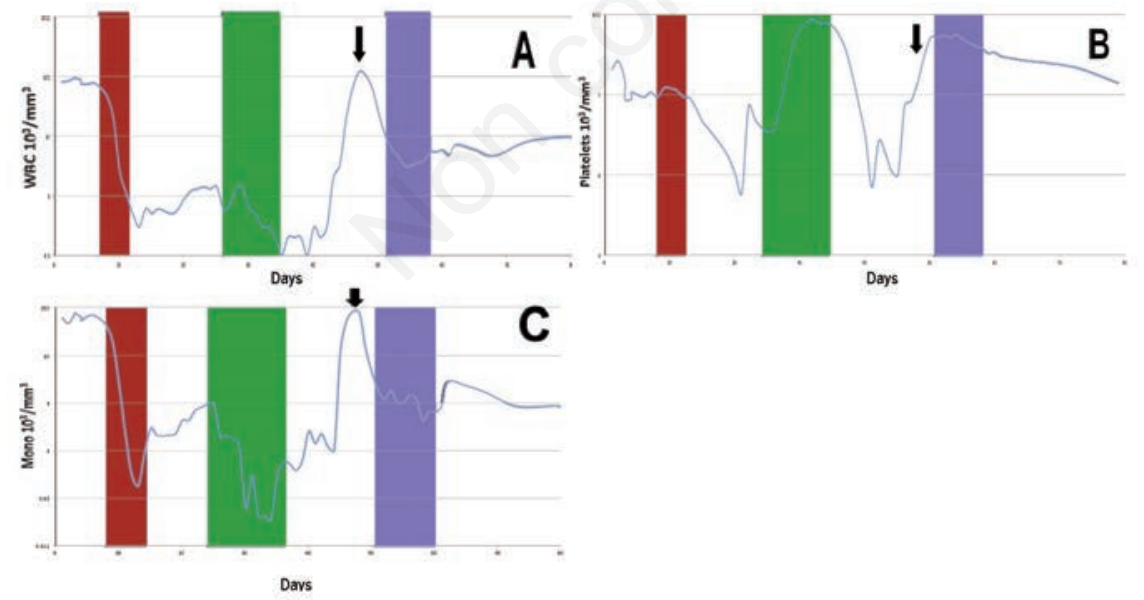

Figure 1. Response of white blood cell (WBC), platelets, and monocytes to chemotherapy with only azacitidine inducing sustainable remission in the first 80 days. The first induction (red box) included fludarabine and cytarabine, and the second induction (green box) included daunorubicin and cytarabine. The WBC normalized only after hydroxyurea (arrow) and the first cycle of azacitidine (blue box) (A). Platelets rose markedly after a brief nadir following the second induction therapy, and only normalized after azacitidine therapy (B). Monocytes (including monoblasts and promonocytes) rebounded following the first two cycles of induction therapy and only normalized after azacitidine therapy (C). chemotherapy reported a $9 \%$ complete response rate following azacitidine based therapy. ${ }^{11}$ Among their cohort with a normal karyotype the one-year survival was $42.5 \%$. These studies underline the efficacy of hypomethylating agents in the treatment of selected patients with relapsed or refractory AML.

Furthermore, combinations of azacitidine with other agents including epigenetic modifiers and other targeted chemotherapeutic agents have shown particular promise. A phase II trial demonstrated increased response rate in MDS with azacitidine and the HDAC inhibitor, vorinostat, compared to historic controls. ${ }^{13}$ Another clinical trial has shown that a combination of azacitidine and the non-specific tyrosine kinase inhibitor sorafenib may be particularly effective in treating relapsed AML with a FLT-3 mutation. ${ }^{14}$ Combination chemotherapy of azacitidine with gemtuzumab ozogamicin for elderly patients not qualifying for intensive induction chemotherapy has achieved a $35 \%$ CR rate in a phase II clinical trial. $^{3}$

Our patient is unusual because of his critical condition at presentation with extreme leukocytosis, hyperuricemia, associated acute renal failure and borderline low cardiac $\mathrm{EF}$. He had cytogenetically normal AML, the most common cytogenetic risk group, which is associated with variable outcomes. ${ }^{15,16}$ While there is little data on association between response to azacitidine and AML with monocytic differentiation, there is evidence that azacitidine is an effective agent in the management of chronic myelomonocytic leukemia. ${ }^{17}$ It would be of interest to investigate further whether a monocytic phenotype is particularly responsive to azacitidine therapy. The aggressiveness of his leukemia is notable as well with the rapid rise of his white blood count to over $100 \times 10^{3} / \mathrm{mm}^{3}$ only two weeks after completing his second induction course. Far more surprising, he developed significant thrombocytosis following two consecutive cycles of induction chemotherapy, a phenomenon observed in the 3q21q26 syndrome, ${ }^{18}$ although he had cytogenetically normal AML. Another possible cause for his thrombocytosis is damage to megakaryocytes after chemotherapy, which explains the newly present atypical megakaryocytes in his bone marrow after the first round of induction chemotherapy. Regardless of the etiology, his platelet count returned to normal levels after azacitidine therapy. Our patient also did not experience the dose-limiting toxicities of azacitidine, such as severe bone marrow suppression and infection. There are only a few reports of patients achieving a complete remission following azacitidine after failing initial remission-induction therapy. ${ }^{6}$

Azacitidine may, in select cases, become a preferred agent for salvage therapy in refracto- 
ry or recurrent AML especially as a bridge to HSCT. ${ }^{10}$ Further prospective clinical trials with a focus on the clinical and molecular behavior are needed to assess further the optimal use of hypomethylating agents in the management of refractory AML.

\section{References}

1. Lowenberg B, Downing JR, Burnett A. Acute myeloid leukemia. N Engl J Med 1999;341:1051-623.

2. Breems DA, Van Putten WL, Huijgens PC, et al. Prognostic index for adult patients with acute myeloid leukemia in first relapse. J Clin Oncol 2005;23:1969-78.

3. Kell WJ, Burnett AK, Chopra R, et al. A feasibility study of simultaneous administration of gemtuzumab ozogamicin with intensive chemotherapy in induction and consolidation in younger patients with acute myeloid leukemia. Blood 2003;102:4277-83.

4. Fernandez HF, Sun Z, Yao X, et al. Anthracycline dose intensification in acute myeloid leukemia. N Engl J Med 2009;361:1249-59.

5. Leopold LH, Willemze R. The treatment of acute myeloid leukemia in first relapse: a comprehensive review of the literature.
Leuk Lymphoma 2002;43:1715-27.

6. Ivanoff S, Gruson B, Chantepie SP, et al. Azacitidine treatment for relapsed or refractory acute myeloid leukemia after intensive chemotherapy. Am J Hematol 2013;88:601-5.

7. Al-Ali HK, Jaekel N, Niederwieser D. The role of hypomethylating agents in the treatment of elderly patients with AML. J Geriatr Oncol 2014;5:85-109.

8. Estey EH. Spotlight on epigenetics in hematological malignancies. Epigenentics in clinical practice: the example of azacytidine and decitabine. Leukemia 2013; 27:1803-12.

9. Von Hoff DD, Slavik M, Muggia FM, et al. 5Azacytidine: a new anticancer drug with effectiveness in acute myelogenous leukemia. Ann Intern Med 1976;85:237-45.

10. Tawfik B, Silesoraitis S, Lyerly S, et al. Efficacy of the hypomethylating agents as frontline, salvage, or consolidation therapy in adults with acute leukemia. Ann Hematol 2014;93:47-55.

11. Itzykson $R$, Thépot $S$, Recher $S$, et al. Azacytidine in refractory or relapsed AML after intensive chemotherapy (IC): results of the French ATU Program. Blood 2009;114:1054.

12. Marbello L, Ricci F, Nosari AM, et al. Outcome of hyperleukocytic adult acute myeloid leukaemia: a single-center retrospective study and review of literature.
Leuk Res 2008;32:1221-27.

13. Silverman L, Verma A, Odchimar-Reissig $\mathrm{R}$, et al. A phase II trial of epigenetic modulators vorinostat with azacitidine in patients with the myelodysplastic syndrome: initial results of study 6898 of the New York cancer consortium. Blood 2013;122:21.

14. Ravandi F, Alattar ML, Grunwald MR, et al. Phase 2 study of azacytidine plus sorafenib in patients with acute myeloid leukemia and FLT-3 internal tandem duplication mutation. Blood 2013;121:4655-62.

15. Ghanem H, Tank N, Tabbara I, et al. Prognostic implications of genetic aberrations in acute myelogenous leukemia with normal cytogenetics. Am J Hematol 2012; 87:69-77.

16. Walker A, Marcucci G. Molecular prognostic factors in cytogenetically normal acute myeloid leukemia. Exp Rev Hematol 2012;5:547-58.

17. Costa C, Abdulhaq H, Haq B, et al. Activity of azacitidine in chronic myelomonocytic leukemia. Cancer 2011;117:2690-96.

18. Bellomo J, Parlier V, Muhlematter D et al. Three new cases of chromosome 3 rearrangement in bands q21 and q26 with abnormal thrombopoiesis bring further evidence to the existence of a 3q21q26 syndrome. Cancer Genet Cytogenet 1992; 59:138-60. 